\title{
Approximation of Functions in Besov Space
}

\author{
Hare Krishna Nigam and Supriya Rani
}

\begin{abstract}
In the present paper, we establish a theorem on best approximation of a function $g \in B_{q}^{\lambda}\left(L^{r}\right)$ of its Fourier series. Our main theorem generalizes some known results of this direction of work. Thus, the results of [10], [26] and [27] become the particular case of our main Theorem 3.1.
\end{abstract}

\section{Introduction}

The degree of approximation of the functions in Lipschitz spaces and Hölder spaces using single and product summability means has been studied by the authors $[7,8,9,15,16,18,19,20,21$, $22,23,25,28]$. This motivates us to study the degree of approximation of a function in more generalized function space. Therefore, in this paper, we study the degree of approximation of a function $g$ in Besov space using Hausdorff-generalized Nörlund means of its Fourier series. It can be noted that Besov space generalizes different Sobolev spaces, Lipschitz spaces and generalized Hölder spaces [13]. Besov space can also be used to study regularity properties of the functions. Let $C_{2 \pi}:=C[0,2 \pi]$ denote the Banach space of all $2 \pi$-periodic continuous functions $g$ defined on $[0,2 \pi]$ under the supremum norm.

Let

$$
L^{r}:=L^{r}[0,2 \pi]:=\left\{g:[0,2 \pi] \mapsto \mathbb{R}: \int_{0}^{2 \pi}|g(z)|^{r} d z<\infty\right\}, r \geq 1
$$

be the space of all $2 \pi$-periodic, integrable functions $L^{r}$-norm of the function $g$ is given by

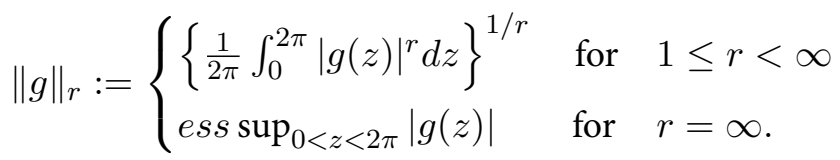

2010 Mathematics Subject Classification. 41A10, 41A25, 42B05, 42A50, 40G05, 40 C05.

Key words and phrases. Degree of approximation; Besov space; Hausdorff $\left(\Delta_{H}\right)$ means; generalized Nörlund $\left(N^{p, q}\right)$ means; Hausdorff-genearlized Nörlund $\left(\Delta_{H} N^{p, q}\right)$ means; Fourier series.

Corresponding author: Hare Krishna Nigam. 
The modulus of continuity of a function $g \in L^{r}$ is defined by

$$
w(g ; l):=\sup _{\substack{z, z+h \in[0,2 \pi] \\|h|<l}}|g(z+h)-g(z)| .
$$

The integral modulus of continuity of the first order of a function $g \in L^{r}$ is defined [3] by

$$
w_{1}(g ; l)_{r}:=\sup _{|h|<l, z \in \mathbb{R}}\|g(z+h)-g(z)\|_{r}
$$

The integral moduli of continuity of the second order (modulus of smoothness) of a function $g \in L^{r}$ is defined [2] by

$$
w_{2}(g ; l)_{r}=\sup _{0<h \leq l, z \in \mathbb{R}}\|g(z+h)+g(z-h)-2 g(z)\|_{r} .
$$

The $j^{t h}$ order modulus of smoothness of a function $g \in L^{r}$ is defined [1] by

$$
w_{j}(g, l)_{r}:=\sup _{0<h \leq l}\left\|\Delta_{h}^{j}(g, \cdot)\right\|_{r}, \quad l>0
$$

where

$$
\Delta_{h}^{j}(g, z):=\sum_{\rho=0}^{j}(-1)^{j-\rho}\left(\begin{array}{l}
j \\
\rho
\end{array}\right) g(z+\rho h), \quad j \in \mathbb{N} .
$$

\section{Remark 1.}

(i) If $r=\infty, j=1$ and $g$ being a continuous function, then $w_{j}(g, l)_{r}$ reduces to $w(g, l)$.

(ii) If $0<r<\infty, j=1$ and $g$ being a continuous function, then $w_{j}(g, l)_{r}$ reduces to $w_{1}(g, l)_{r}$.

(iii) If $g \in C_{2 \pi}$ and $w(g, l)=O\left(l^{\lambda}\right), 0<\lambda \leq 1$, then $g \in \operatorname{Lip} \lambda$.

(iv) If $g \in L^{r}, 0<r<\infty$ and $w(g, l)_{r}=O\left(l^{\lambda}\right), 0<\lambda \leq 1$, then $g \in \operatorname{Lip}(\lambda, r)$.

(v) If $r=\infty$, then $\operatorname{Lip}(\lambda, r)$ class reduces to Lip $\lambda$.

Note 1. From Remark 1(iv) and 1(v), we write

$$
\operatorname{Lip}(\lambda) \subseteq \operatorname{Lip}(\lambda, r)
$$

Let $\lambda>0, j>\lambda$ i.e. $j=[\lambda]+1$, where $j$ being smallest integer. For $g \in L^{r}$, if

$$
w_{j}(g, l)_{r}=O\left(l^{\lambda}\right), \quad l>0
$$


then $g \in \operatorname{Lip}^{*}(\lambda, r)$ and its semi-norm is given by

$$
|g|_{L i p^{*}(\lambda, r)}=\sup _{l>0}\left(l^{-\lambda} w_{j}(g, l)_{r}\right),
$$

where $\operatorname{Lip}^{*}(\lambda, r)$ is a generalized Lipschitz class of function $g$.

Thus,

$$
\operatorname{Lip}(\lambda, r) \subseteq \operatorname{Lip}^{*}(\lambda, r)
$$

For $0<\lambda \leq 1$, let

$$
H_{\lambda}:=\left\{g \in C_{2 \pi}: w(g, l)=O\left(l^{\lambda}\right)\right\}
$$

where $H_{\lambda}$ is a Banach space with the norms

$$
\|g\|_{\lambda}=\|g\|_{C}+\sup _{l>0}\left(l^{-\lambda} w(l)\right) \quad \text { for } \quad 0<\delta \leq \lambda<1
$$

and

$$
\|g\|_{0}=\|g\|_{C}
$$

Thus, we observe that

$$
H_{\lambda} \subseteq H_{\delta} \subseteq C_{2 \pi} \text { for } \quad 0<\delta \leq \alpha<1([28])
$$

The metric induced by the norm $\|\cdot\|_{\lambda}$ on $H_{\lambda}$ is called the Hölder metric.

For $0<\lambda \leq 1,0<r \leq \infty$, let

$$
H_{\lambda, r}:=H_{\lambda, r}[0,2 \pi]=\left\{g \in L^{r}: w(g, l)_{r}=O\left(l^{\lambda}\right)\right\}
$$

where $H_{\lambda, r}$ is also a Banach space with the norm $\|\cdot\|_{\lambda, r}$ defined by

$$
\|g\|_{\lambda, r}=\|g\|_{r}+\sup _{l>0}\left(l^{-\lambda} w(g, l)_{r}\right) \text { for } 0<\lambda \leq 1
$$

and

$$
\|g\|_{0, r}=\|g\|_{r}
$$

Then $H_{\lambda, r}$ is a Banach space for $r \geq 1$ and a complete $r$-normed space ([14], p. 87) for $0<r<1$.

Thus,

$$
H_{\lambda, r} \subseteq H_{\delta, r} \subseteq L^{r} \quad \text { for } \quad 0<\delta \leq \lambda \leq 1([9])
$$


For $\lambda>0$ and let $j>\lambda$ i.e. $j=[\lambda]+1$. For $0<r, q \leq \infty$, the Besov space $B_{q}^{\lambda}\left(L^{r}\right)$ is a collection of all $2 \pi$-periodic function $g \in L^{r}$ such that

$$
|g|_{B_{q}^{\lambda}\left(L^{r}\right)}:=\left\|w_{j}(g, \cdot)\right\|_{\lambda, q}= \begin{cases}\left(\int_{0}^{\pi}\left[l^{-\lambda} w_{j}(g, l)_{r}\right]^{q} \frac{d l}{l}\right)^{\frac{1}{q}}, & 0<q<\infty \\ \sup _{l>0}\left(l^{-\lambda} w_{j}(g, l)_{r}\right), & q=\infty\end{cases}
$$

is finite ([24], p. 237) for $2 \pi$ - periodic function $g$ ([1], p. 54).

It is further observed that (1.7) is a semi-norm if $1 \leq r, q \leq \infty$ and a quasi semi-norm in other cases ([1], p. 55).

The quasi-norm for Besov space is given by

$$
\begin{aligned}
\|g\|_{B_{q}^{\lambda}\left(L^{r}\right)}: & =\|g\|_{r}+|g|_{B_{q}^{\lambda}\left(L^{r}\right)} \\
& =\|g\|_{r}+\left\|w_{j}(g, \cdot)\right\|_{\lambda, q}
\end{aligned}
$$

\section{Note 2.}

(i) If $0<\lambda<1$, then the Besov space $B_{\infty}^{\lambda}\left(L^{r}\right)$ reduces to the $H_{\lambda, r}$ ([9]).

(ii) If $r=\infty=q$ and $0<\lambda<1$, the Besov space $B_{\infty}^{\lambda}\left(L^{r}\right)$ reduces to the space $H_{\lambda}$ ([28]).

The $m$-order error approximation of a function $g \in C_{2 \pi}$ is defined by $E_{m}(g):=\inf _{t_{m}}\left\|g-t_{m}\right\|$ where $t_{m}$ is a trigonometric polynomial of degree $m$ ([3]).

If $E_{m}(g) \rightarrow 0$ as $m \rightarrow \infty, E_{m}(g)$ is said to be the best approximation of $g$ ([3]).

\section{Definitions}

The Hausdorff matrix $H \equiv\left(h_{m, j}\right)$ is an infinite lower triangular matrix defined by,

$$
h_{m, j}= \begin{cases}\left(\begin{array}{c}
m \\
j
\end{array}\right) \Delta^{m-j} \mu_{j}, & 0 \leq j \leq m, \\
0, & j>m,\end{cases}
$$

where $\Delta$ is a forward operator defined by $\Delta \mu_{m}=\mu_{m}-\mu_{m+1}$ and $\Delta^{j+1} \mu_{m}=\Delta\left(\Delta^{j} \mu_{m}\right)([6])$. A Hausdorff matrix $H$ is regular iff $\int_{0}^{1}|d \gamma(y)|<\infty$, where the mass function $\gamma(y)$ is continuous at $y=0$ and belongs to $\mathrm{BV}[0,1]$ such that $\gamma(0+)=0, \gamma(1)=1$; and for $0<y<1, \gamma(y)=$ $[\gamma(y+0)+\gamma(y-0)] / 2[4,11]$. Thus $\left\{\mu_{m}\right\}$, known as moment sequence, has the representation

$$
\mu_{m}=\int_{0}^{1} y^{m} d \gamma(y)
$$


The Hausdorff means of a trigonometric Fourier series of $g$ is defined by

$$
H_{m}(g ; z)=\sum_{j=0}^{m} h_{m, j} s_{j}(g ; z), \quad \forall \quad m \geq 0 .
$$

The series is said to be summable to $s$ by Hausdorff means, if $H_{m}(g ; z) \rightarrow s$ as $m \rightarrow \infty$ and we denote Hausdorff means by $\Delta_{H}$ through out the paper.

Example 1. (i) If

$$
h_{m, j}= \begin{cases}\left(\begin{array}{c}
m \\
j
\end{array}\right) \frac{q^{m-j}}{(1+q)^{m}}, & 0 \leq j \leq m, \\
0, & j>m,\end{cases}
$$

then the Hausdorff matrix $H \equiv\left(h_{m, j}\right)$ reduces to $(E, q)$ matrix (Euler matrix of order $q>0)$ and defines the corresponding $(E, q)$ means by

$$
E_{m}^{q}(g ; z):=\frac{1}{(1+q)^{m}} \sum_{j=0}^{m}\left(\begin{array}{c}
m \\
j
\end{array}\right) q^{m-j} s_{j}(g ; z) .
$$

(ii) If $\mu_{m}=\frac{1}{m+1}$ then the Hausdorff matrix $H \equiv\left(h_{m, j}\right)$ reduces to $(C, 1)$ matrix (Cesàro matrix of order 1) and defines the corresponding means by

$$
H_{m}(g ; z):=\frac{1}{(m+1)} \sum_{j=0}^{m} s_{j}(g ; z) .
$$

Let $\left\{p_{m}\right\}$ and $\left\{q_{m}\right\}$ be the sequence of constants, real or complex, such that

$$
\begin{aligned}
& P_{m}=p_{0}+p_{1}+p_{2}+\cdots+p_{m}=\sum_{v=0}^{m} p_{v} \rightarrow \infty, \quad \text { as } \quad m \rightarrow \infty \\
& Q_{m}=q_{0}+q_{1}+q_{2}+\cdots+q_{m}=\sum_{v=0}^{m} q_{v} \rightarrow \infty, \quad \text { as } \quad m \rightarrow \infty \\
& R_{m}=p_{0} q_{m}+p_{1} q_{m-1}+p_{2} q_{m-2}+\cdots+p_{m} q_{0}=\sum_{v=0}^{m} p_{v} q_{m-v} \rightarrow \infty, \quad \text { as } \quad m \rightarrow \infty .
\end{aligned}
$$

Given two sequences $\left\{p_{m}\right\}$ and $\left\{q_{m}\right\}$ convolution $(p * q)$ is defined as

$$
R_{m}=(p * q)_{m}=\sum_{j=0}^{m} p_{m-j} q_{j}
$$

We write

$$
t_{m}^{p, q}=\frac{1}{R_{m}} \sum_{j=0}^{m} p_{m-j} q_{j} s_{j}
$$


If $R_{m} \neq 0$, for all $m$, the generalized Nörlund transform of the sequence $\left\{s_{m}\right\}$ is the sequence $\left\{t_{m}^{p, q}\right\}$. If $t_{m}^{p, q} \rightarrow s$, as $m \rightarrow \infty$, then the series $\sum_{m=0}^{\infty} a_{m}$ or sequence $\left\{s_{m}\right\}$ is summable to $s$ by generalized Nörlund method and is denoted by $s_{m} \rightarrow s\left(N^{p, q}\right)$.

The necessary and sufficient condition for $\left(N^{p, q}\right)$ method to be regular are

$$
\sum_{j=0}^{m}\left|p_{m-j} q_{j}\right|=O\left(\left|R_{m}\right|\right)
$$

and $p_{m-j}=o\left(\left|R_{m}\right|\right)$, as $m \rightarrow \infty$ for every fixed $j \geq 0$, for which $q_{j} \neq 0$ ([17]).

If the method $\Delta_{H}$ is superimposed on the $N^{p, q}$ method, another new method of summability $\Delta_{H} N^{p, q}$ is obtained.

The Hausdorff transform of $N^{p, q}$ transform is defined as $\Delta_{H} N^{p, q}$ product transform of the partial sum $s_{m}$, which can be given by

$$
\begin{aligned}
t_{m}^{\Delta_{H} N^{p, q}} & =\sum_{j=0}^{m} h_{m, j} t_{j}^{p, q} \\
& =\sum_{j=0}^{m} h_{m, j} \frac{1}{R_{j}} \sum_{v=0}^{j} p_{j-v} q_{v} s_{v} .
\end{aligned}
$$

If $t_{m}^{\Delta_{H} N^{p, q}} \rightarrow s$ as $m \rightarrow \infty$ then the series $\sum_{m=0}^{\infty} a_{m}$ or the sequence $\left\{s_{m}\right\}$ is summable to s by $\Delta_{H} N^{p, q}$ means.

Now, we define the regularity of $\Delta_{H} N^{p, q}$ method.

$$
\begin{aligned}
s_{m} \rightarrow s & \Longrightarrow t_{m}^{p, q} \rightarrow s, \quad \text { as } m \rightarrow \infty \quad \text { so } N^{p, q} \text { method is regular, } \\
& \Longrightarrow \Delta\left(t_{m}^{p, q}\right)=t_{m}^{\Delta_{H} N^{p, q}} \rightarrow s \quad \text { as } m \rightarrow \infty \text { so } \Delta_{H} \text { method is regular, } \\
& \Longrightarrow\left(\Delta_{H} N^{p, q}\right) \quad \text { method is regular. }
\end{aligned}
$$

\section{Remark 2.}

(i) $\Delta_{H} N^{p, q}$ means reduces to $E_{q} N^{p, q}$ means if $h_{m, j}= \begin{cases}\left(\begin{array}{c}m \\ j\end{array}\right) \frac{q^{m-j}}{(1+q)^{m}}, & \text { if } 0 \leq j \leq m, \\ 0, & \text { if } j>m .\end{cases}$

(ii) $\Delta_{H} N^{p, q}$ means reduces to $C_{1} N^{p, q}$ means if $h_{m, j}= \begin{cases}\frac{1}{m+1}, & \text { if } 0 \leq j \leq m, \\ 0, & \text { if } j>m .\end{cases}$

(iii) $\Delta_{H} N^{p, q}$ means reduces to $\Delta_{H} N^{p_{m}}$ means if $q_{m}=1, \forall m$.

(iv) $\Delta_{H} N^{p, q}$ means reduces to $\Delta_{H} \tilde{N}^{q_{m}}$ means if $p_{m}=1, \forall m$. 
(v) $\Delta_{H} N^{p, q}$ means reduces to $\Delta_{H} C_{\alpha}$ means if $p_{m}=\left(\begin{array}{c}m+\alpha-1 \\ \alpha-1\end{array}\right), \alpha>0$ and $q_{m}=1, \forall m$.

Remark 3. The above cases (i) and (ii) of Remark 2 can be further reduced as

(i) $E_{q} N^{p, q}$ means reduces to $E_{q} N^{p_{m}}$ means if $q_{m}=1, \forall m$.

(ii) $E_{q} N^{p, q}$ means reduces to $E_{1} N^{p_{m}}$ means if $q_{m}=1, \forall m$.

(iii) $E_{q} N^{p, q}$ means reduces to $E_{q} \tilde{N}^{q_{m}}$ means if $p_{m}=1, \forall m$.

(iv) $E_{q} N^{p, q}$ means reduces to $E_{q} C_{\alpha}$ means if $p_{m}=\left(\begin{array}{c}m+\alpha-1 \\ \alpha-1\end{array}\right), \alpha>0$ and $q_{m}=1, \forall m$.

(v) $C_{1} N^{p, q}$ means reduces to $C_{1} N^{p_{m}}$ means if $q_{m}=1, \forall m$.

(vi) $C_{1} N^{p, q}$ means reduces to $C_{1} \tilde{N}^{q_{m}}$ means if $p_{m}=1, \forall m$.

\section{Main Theorems}

Theorem 3.1. If $g$ is a $2 \pi$-periodic and Lebesgue integrable function, then for $0 \leq \delta<\lambda<2$, the best approximation of $g$ in $B_{q}^{\lambda}\left(L^{r}\right), r \geq 1,1<q \leq \infty$ space using $\Delta_{H} N^{p, q}$ means, is given by $\left\|t_{m}^{\Delta_{H} N^{p, q}}(z)-g(z)\right\|= \begin{cases}O(m+1)^{-\lambda}+O\left(\frac{1}{(m+1)^{\lambda-\delta-(1 / q)}}\right)+O\left(\frac{1}{(m+1)^{\lambda-\delta}}\right) & ; 1<q<\infty \\ O(m+1)^{-\lambda}+O\left(\frac{1}{(m+1)^{\lambda-\delta}}\right) & ; q=\infty .\end{cases}$

\section{Lemmas}

Lemma 4.1. Let $K_{m}^{\Delta_{H} N^{p, q}}(\eta):=\int_{0}^{1} M_{m}(\eta) d \gamma(y)$

where

$$
M_{m}(\eta):=\left[\sum_{j=0}^{m}\left(\begin{array}{c}
m \\
j
\end{array}\right) y^{j}(1-y)^{m-j}\left\{\frac{1}{R_{j}} \sum_{\nu=0}^{j} p_{j-\nu} q_{\nu} \frac{\sin \left(\nu+\frac{1}{2}\right) \eta}{2 \sin \frac{\eta}{2}}\right\}\right]
$$

then

$$
K_{m}^{\Delta_{H} N^{p, q}}(\eta)= \begin{cases}O(m+1), & 0 \leq \eta \leq \frac{1}{(m+1)} \\ O\left(\frac{1}{\eta}\right), & \frac{1}{(m+1)} \leq \eta \leq \pi\end{cases}
$$


Proof. For $0 \leq \eta \leq \frac{1}{m+1}$, we have $\sin m \eta \leq m \sin \eta$, then

$$
\begin{aligned}
& M_{m}(\eta) \\
& =\left[\sum_{j=0}^{m}\left(\begin{array}{c}
m \\
j
\end{array}\right) y^{j}(1-y)^{m-j}\left\{\frac{1}{R_{j}} \sum_{\nu=0}^{j} p_{j-\nu} q_{\nu} \frac{\sin \left(\nu+\frac{1}{2}\right) \eta}{2 \sin \frac{\eta}{2}}\right\}\right] \\
& \leq \frac{1}{2}\left[\sum_{j=0}^{m}\left(\begin{array}{c}
m \\
j
\end{array}\right) y^{j}(1-y)^{m-j}\left\{\frac{1}{R_{j}} \sum_{\nu=0}^{j} p_{j-\nu} q_{\nu} \frac{(2 \nu+1) \sin \frac{\eta}{2}}{\sin \frac{\eta}{2}}\right\}\right] \\
& \leq \frac{1}{2}\left[\sum_{j=0}^{m}\left(\begin{array}{c}
m \\
j
\end{array}\right) y^{j}(1-y)^{m-j}\left\{\frac{1}{R_{j}} \sum_{\nu=0}^{j} p_{j-\nu} q_{\nu}(2 \nu+1)\right\}\right] \\
& =\frac{1}{2}\left[\sum_{j=0}^{m}\left(\begin{array}{c}
m \\
j
\end{array}\right) y^{j}(1-y)^{m-j} \frac{1}{R_{j}}\left\{p_{j} q_{0}(2 \cdot 0+1)+p_{j-1} q_{1}(2 \cdot 1+1)+\cdots+p_{0} q_{j}(2 \cdot j+1)\right\}\right] \\
& \leq \frac{1}{2}\left[\sum_{j=0}^{m}\left(\begin{array}{c}
m \\
j
\end{array}\right) y^{j}(1-y)^{m-j} \frac{1}{R_{j}}\left\{p_{j} q_{0}(2 j+1)+p_{j-1} q_{1}(2 j+1)+\cdots+p_{0} q_{j}(2 j+1)\right\}\right] \\
& =\frac{1}{2}\left[\sum_{j=0}^{m}\left(\begin{array}{c}
m \\
j
\end{array}\right) y^{j}(1-y)^{m-j} \frac{1}{R_{j}}(2 j+1) \sum_{\nu=0}^{j} p_{j-\nu} q_{\nu}\right] \\
& =\frac{1}{2}\left[\sum_{j=0}^{m}\left(\begin{array}{c}
m \\
j
\end{array}\right) y^{j}(1-y)^{m-j} \frac{1}{R_{j}}(2 j+1) O\left(\left|R_{j}\right|\right)\right] \\
& =O\left[\sum_{j=0}^{m}\left(\begin{array}{c}
m \\
j
\end{array}\right) y^{j}(1-y)^{n-j}(2 j+1)\right] \\
& =O\left[2 \sum_{j=0}^{m}\left(\begin{array}{c}
m \\
j
\end{array}\right) y^{j}(1-y)^{n-j} \cdot j+\sum_{j=0}^{m}\left(\begin{array}{c}
m \\
j
\end{array}\right) y^{j}(1-y)^{m-j}\right] .
\end{aligned}
$$

Now, solving first term of (4.1),

$$
\begin{aligned}
\sum_{j=0}^{m}\left(\begin{array}{c}
m \\
j
\end{array}\right) y^{j}(1-y)^{m-j} \cdot j & =(1-y)^{m} \sum_{j=0}^{m}\left(\begin{array}{c}
m \\
j
\end{array}\right)\left(\frac{y}{1-y}\right)^{j} \cdot j \\
& =(1-y)^{m} \sum_{j=0}^{m}\left(\begin{array}{c}
m \\
j
\end{array}\right) p^{j} \cdot j,
\end{aligned}
$$

where $\frac{y}{1-y}=p \Rightarrow 1+p=\frac{1}{1-y}$.

$$
\sum_{j=0}^{m}\left(\begin{array}{c}
m \\
j
\end{array}\right) p^{j} \cdot j=\left(\begin{array}{c}
m \\
0
\end{array}\right) p^{0} \cdot 0+\left(\begin{array}{c}
m \\
1
\end{array}\right) p^{1} \cdot 1+\left(\begin{array}{c}
m \\
2
\end{array}\right) p^{2} \cdot 2+\cdots+\left(\begin{array}{c}
m \\
m
\end{array}\right) p^{m} \cdot m
$$




$$
=\left(\begin{array}{c}
m \\
1
\end{array}\right) p+2\left(\begin{array}{c}
m \\
2
\end{array}\right) p^{2}+3\left(\begin{array}{c}
m \\
3
\end{array}\right) p^{3}+\cdots+m\left(\begin{array}{c}
m \\
m
\end{array}\right) p^{m}
$$

We know that

$$
(1+p)^{m}=\left(\begin{array}{c}
m \\
0
\end{array}\right)+\left(\begin{array}{c}
m \\
1
\end{array}\right) \cdot p+\left(\begin{array}{c}
m \\
2
\end{array}\right) \cdot p^{2}+\cdots+\left(\begin{array}{c}
m \\
m
\end{array}\right) \cdot p^{m} .
$$

By diffrentiating with respect to $p$, we have

$$
m(1+p)^{m-1}=0+\left(\begin{array}{c}
m \\
1
\end{array}\right)+\left(\begin{array}{c}
m \\
2
\end{array}\right) \cdot 2 p+\left(\begin{array}{c}
m \\
3
\end{array}\right) \cdot 3 p^{2}+\cdots+\left(\begin{array}{c}
m \\
m
\end{array}\right) \cdot m p^{m-1} .
$$

Multiplying above equation by $p$ on both side, we have

$$
m p(1+p)^{m-1}=\left(\begin{array}{c}
m \\
1
\end{array}\right) p+2\left(\begin{array}{c}
m \\
2
\end{array}\right) p^{2}+3\left(\begin{array}{c}
m \\
3
\end{array}\right) p^{3}+\cdots+m\left(\begin{array}{c}
m \\
m
\end{array}\right) p^{m} .
$$

Now, from (4.2) and (4.3), we have

$$
\begin{gathered}
\sum_{j=0}^{m}\left(\begin{array}{c}
m \\
j
\end{array}\right)\left(\frac{y}{1-y}\right)^{j} \cdot j=m p(1+p)^{m-1} \\
(1-y)^{m} \sum_{j=0}^{m}\left(\begin{array}{c}
m \\
j
\end{array}\right)\left(\frac{y}{1-y}\right)^{j} \cdot j=(1-y)^{m}\left\{m p(1+p)^{m-1}\right\} \\
\sum_{j=0}^{m}\left(\begin{array}{c}
m \\
j
\end{array}\right) y^{j}(1-y)^{m-j} \cdot j=(1-y)^{m}\left\{m \cdot \frac{y}{1-y} \cdot \frac{1}{(1-y)^{m-1}}\right\} \\
\sum_{j=0}^{m}\left(\begin{array}{c}
m \\
j
\end{array}\right) y^{j}(1-y)^{m-j} \cdot j=m y .
\end{gathered}
$$

Now, solving second term of (4.1),

$$
\begin{aligned}
\sum_{j=0}^{m} & \left(\begin{array}{c}
m \\
j
\end{array}\right) y^{j}(1-y)^{m-j} \\
& =\left(\begin{array}{c}
m \\
0
\end{array}\right) y^{0}(1-y)^{m}+\left(\begin{array}{c}
m \\
1
\end{array}\right) y^{1}(1-y)^{m-1}+\cdots+\left(\begin{array}{c}
m \\
m
\end{array}\right) y^{m}(1-y)^{m-m} \\
& =(1-y+y)^{m} \\
& =1 .
\end{aligned}
$$

Now, from (4.1), (4.4) and (4.5), we get

$$
M_{m}(\eta)=O(2 m y+1) .
$$


Thus,

$$
\begin{aligned}
K_{m}^{\Delta_{H} N^{p, q}}(\eta) & =\int_{0}^{1} M_{m}(\eta) d \gamma(y) \\
& =O(1) \int_{0}^{1}(2 m y+1) d y \\
& =O(m+1) .
\end{aligned}
$$

For $\frac{1}{m+1} \leq \eta \leq \pi$, by Jordan's lemma we have, $\sin \frac{\eta}{2} \geq \frac{\eta}{\pi}$ and $\sin n \eta \leq 1$. Thus,

$$
\begin{aligned}
M_{m}(\eta) & =\left[\sum_{j=0}^{m}\left(\begin{array}{c}
m \\
j
\end{array}\right) y^{j}(1-y)^{m-j}\left\{\frac{1}{R_{j}} \sum_{\nu=0}^{j} p_{j-\nu} q_{\nu} \frac{\sin \left(\nu+\frac{1}{2}\right) \eta}{2 \sin \frac{\eta}{2}}\right\}\right] \\
& \leq \frac{1}{2}\left[\sum_{j=0}^{m}\left(\begin{array}{c}
m \\
j
\end{array}\right) y^{j}(1-y)^{m-j}\left\{\frac{1}{R_{j}} \sum_{\nu=0}^{j} p_{j-\nu} q_{\nu} \frac{1}{\frac{\eta}{\pi}}\right\}\right] \\
& \leq \frac{\pi}{2 \eta}\left[\sum_{j=0}^{m}\left(\begin{array}{c}
m \\
j
\end{array}\right) y^{j}(1-y)^{m-j}\left\{\frac{1}{R_{j}} \sum_{\nu=0}^{j} p_{j-\nu} q_{\nu}\right\}\right] \\
& =\frac{\pi}{2 \eta}\left[\begin{array}{l}
m \\
j=0
\end{array}\left(\begin{array}{c}
m \\
j
\end{array}\right) y^{j}(1-y)^{m-j}\left\{\frac{1}{R_{j}} O\left(\left|R_{j}\right|\right)\right\}\right] \\
& =\frac{\pi}{2 \eta}\left[\sum_{j=0}^{m}\left(\begin{array}{c}
m \\
j
\end{array}\right) y^{j}(1-y)^{m-j}\{O(1)\}\right] \\
& =O\left(\frac{\pi}{2 \eta}\right)[\begin{array}{l}
m \\
j=0
\end{array} \underbrace{m}_{j} y^{j}(1-y)^{m-j}] \\
& =O\left(\frac{1}{\eta}\right) \operatorname{since}_{j=0}^{m}\left(\begin{array}{c}
m \\
j
\end{array}\right) y^{j}(1-y)^{m-j}=1 .
\end{aligned}
$$

Thus,

$$
\begin{aligned}
K_{m}^{\Delta_{H} N^{p, q}}(\eta) & =\int_{0}^{1} M_{m}(\eta) d \gamma(y) \\
& =\int_{0}^{1} O\left(\frac{1}{\eta}\right) d y \\
& =O\left(\frac{1}{\eta}\right) \int_{0}^{1} d y \\
& =O\left(\frac{1}{\eta}\right)
\end{aligned}
$$


Lemma 4.2. ([12]) Let $1 \leq r \leq \infty$ and $0<\lambda<2$. If $g \in L^{r}$ then for $0<l$, $\eta \leq \pi$ :

(i) $\|\Phi(\cdot, l, \eta)\|_{r} \leq 4 w_{j}(g, l)_{r}$,

(ii) $\|\Phi(\cdot, l, \eta)\|_{r} \leq 4 w_{j}(g, \eta)_{r}$,

(iii) $\|\Phi(\eta)\|_{r} \leq 2 w_{j}(g, \eta)_{r}$,

where $j=[\lambda]+1$.

Lemma 4.3. Let $0 \leq \delta<\lambda<2$. If $g \in B_{q}^{\lambda}\left(L^{r}\right), r \geq 1,1<q<\infty$, then

$$
\begin{aligned}
& \text { (i) } \int_{0}^{\pi}\left|K_{m}^{\Delta_{H} N^{p, q}}(\eta)\right|\left(\int_{0}^{\eta} \frac{\|\Phi(\cdot, l, \eta)\|_{r}^{q}}{l \delta q} \frac{d l}{l}\right)^{\frac{1}{q}} d \eta= \\
& O(1)\left\{\int_{0}^{\pi}\left(\eta^{\lambda-\delta}\left|K_{m}^{\Delta_{H} N^{p, q}}(\eta)\right|\right)^{\frac{q}{q-1}} d \eta\right\}^{1-(1 / q)}, \\
& \text { (ii) } \int_{0}^{\pi}\left|K_{m}^{\Delta_{H} N^{p, q}}(\eta)\right|\left(\int_{\eta}^{\pi} \frac{\|\Phi(\cdot, l, \eta)\|_{r}^{q}}{l \delta q} \frac{d l}{l}\right)^{\frac{1}{q}} d \eta= \\
& O(1)\left\{\int_{0}^{\pi}\left(\eta^{\lambda-\delta+(1 / q)}\left|K_{m}^{\Delta_{H} N^{p, q}}(\eta)\right|\right)^{\frac{q}{q-1}} d \eta\right\}^{1-(1 / q)} .
\end{aligned}
$$

Proof. This Lemma can be proved along the same lines of the proof of Lemma 1 of [12].

Lemma 4.4. ([12]) Let $0 \leq \delta<\lambda<2$. If $g \in B_{q}^{\lambda}\left(L^{r}\right), r \geq 1, q=\infty$, then

$$
\sup _{0<l, \eta \leq \pi}\left(l^{-\delta}\|\Phi(\cdot, l, \eta)\|_{r}\right)=O\left(\eta^{\lambda-\delta}\right) .
$$

\section{Proof of the Main theorem}

Proof. Following [5], $s_{m}(g, z)$ of Fourier series is given by

$$
s_{m}(g ; z)-g(z)=\frac{1}{2 \pi} \int_{0}^{\pi} \phi_{z}(\eta) \frac{\sin \left(m+\frac{1}{2}\right) \eta}{\sin \frac{\eta}{2}} d \eta .
$$

Denoting the $N^{p, q}$ summability transform of $s_{m}(g ; z)$ by $t_{m}^{p, q}(z)$, we get

$$
t_{m}^{p, q}(z)-g(z)=\sum_{j=0}^{m} t_{j}^{p, q}\left\{s_{j}(g ; z)-g(z)\right\}
$$




$$
\begin{aligned}
& =\frac{1}{\pi} \int_{0}^{\pi}\left(\phi_{z}(\eta) \sum_{j=0}^{m} t_{j}^{p, q} \frac{\sin \left(m+\frac{1}{2}\right) \eta}{2 \sin \frac{\eta}{2}}\right) d \eta \\
& =\left\{\frac{1}{2 \pi} \int_{0}^{\pi} \phi_{z}(\eta) \sum_{j=0}^{m}\left(\frac{1}{R_{j}} \sum_{\nu=0}^{j} p_{j-\nu} q_{\nu} \frac{\sin \left(m+\frac{1}{2}\right) \eta}{\sin \frac{\eta}{2}}\right)\right\} d \eta .
\end{aligned}
$$

The Hausdorff transform of $t_{m}^{p, q}(z)$ i.e., $\Delta_{H} N^{p, q}$ transform of $s_{m}(g ; z)$ denoted by $t_{m}^{\Delta_{H} N^{p, q}}$, is given by

$$
\begin{aligned}
& t_{m}{ }^{\Delta_{H} N^{p, q}}(z)-g(z) \\
& =\sum_{j=0}^{m} h_{m, j}\left\{t_{m}^{p, q}(z)-g(z)\right\} \\
& =\sum_{j=0}^{m}\left(\begin{array}{c}
m \\
j
\end{array}\right) \Delta^{m-j} \mu_{j}\left\{t_{m}^{p, q}(z)-g(z)\right\} \\
& =\sum_{j=0}^{m}\left(\begin{array}{c}
m \\
j
\end{array}\right) \Delta^{m-j} \mu_{j}\left\{\frac{1}{R_{j}} \sum_{\nu=0}^{j} p_{j-\nu} q_{\nu} s_{\nu}\right\} \\
& =\frac{1}{\pi} \int_{0}^{\pi} \phi_{z}(\eta) \sum_{j=0}^{m}\left(\begin{array}{c}
m \\
j
\end{array}\right) \Delta^{m-j} \mu_{j}\left\{\frac{1}{R_{j}} \sum_{\nu=0}^{j} p_{j-\nu} q_{\nu} \frac{\sin \left(m+\frac{1}{2}\right) \eta}{2 \sin \frac{\eta}{2}}\right\} d \eta \\
& =\frac{1}{2 \pi} \int_{0}^{\pi} \phi_{z}(\eta) \sum_{j=0}^{m}\left(\begin{array}{c}
m \\
j
\end{array}\right) \int_{0}^{1} y^{j}(1-y)^{m-j} d \gamma(y)\left\{\frac{1}{R_{j}} \sum_{\nu=0}^{j} p_{j-\nu} q_{\nu} \frac{\sin \left(m+\frac{1}{2}\right) \eta}{\sin \frac{\eta}{2}}\right\} d \eta \\
& =\frac{1}{2 \pi} \int_{0}^{\pi} \phi_{z}(\eta)\left\{\int_{0}^{1} \sum_{j=0}^{m}\left(\begin{array}{c}
m \\
j
\end{array}\right) y^{j}(1-y)^{m-j} \frac{1}{R_{j}} \sum_{\nu=0}^{j} p_{j-\nu} q_{\nu} \frac{\sin \left(m+\frac{1}{2}\right) \eta}{\sin \frac{\eta}{2}} d \gamma(y)\right\} d \eta \\
& =\int_{0}^{\pi} \phi_{z}(\eta) K_{m}^{\Delta_{H} N^{p, q}}(\eta) d \eta .
\end{aligned}
$$

Let

$$
l_{m}(z):=t_{m}^{\Delta_{H} N^{p, q}}(z)-g(z)=\frac{1}{\pi} \int_{0}^{\pi} \phi_{z}(\eta) K_{m}^{\Delta_{H} N^{p, q}}(\eta) d \eta
$$

where

$$
\begin{aligned}
K_{m}^{\Delta_{H} N^{p, q}}(\eta) & =\int_{0}^{1} \sum_{j=0}^{m}\left(\begin{array}{c}
m \\
j
\end{array}\right) y^{j}(1-y)^{m-j}\left\{\frac{1}{R_{j}} \sum_{\nu=0}^{j} p_{j-\nu} q_{\nu} \frac{\sin \left(m+\frac{1}{2}\right) \eta}{\sin \frac{\eta}{2}}\right\} d \gamma(y) \\
& =\int_{0}^{1} M_{m}(\eta) d \gamma(y),
\end{aligned}
$$

where

$$
M_{m}(\eta)=\sum_{j=0}^{m}\left(\begin{array}{c}
m \\
j
\end{array}\right) y^{j}(1-y)^{m-j}\left\{\frac{1}{R_{j}} \sum_{\nu=0}^{j} p_{j-\nu} q_{\nu} \frac{\sin \left(m+\frac{1}{2}\right) \eta}{\sin \frac{\eta}{2}}\right\} .
$$


We write,

$$
\Phi(z, l, \eta)= \begin{cases}\phi_{z+l}(\eta)-\phi_{z}(\eta), & 0<\lambda<1 \\ \phi_{z+l}(\eta)+\phi_{z-l}(\eta)-2 \phi_{z}(\eta), & 1 \leq \lambda<2\end{cases}
$$

and

$$
\mathcal{L}_{m}(z, l)= \begin{cases}l_{m}(z+l)-l_{m}(z), & 0<\lambda<1 \\ l_{m}(z+l)+l_{m}(z-l)-2 l_{m}(z), & 1 \leq \lambda<2 .\end{cases}
$$

Now, we have

$$
\mathcal{L}_{m}(z, l)=\frac{1}{\pi} \int_{0}^{\pi} K_{m}^{\Delta_{H} N^{p, q}}(\eta) \Phi(z, l, \eta) d \eta \quad \text { and } \quad \omega_{j}\left(l_{m}, l\right)_{r}=\left\|\mathcal{L}_{m}(\cdot, l)\right\|_{r} .
$$

Case I : For $1<q<\infty, r \geq 1,0 \leq \delta<\lambda<2$.

By definition, we have

$$
\left\|l_{m}(\cdot)\right\|_{B_{q}^{\delta}\left(L^{r}\right)}=\left\|l_{m}(\cdot)\right\|_{r}+\left\|w_{j}\left(l_{m}, \cdot\right)\right\|_{\delta, q} \cdot
$$

Using generalized Minkowski’s inequality [3], Lemma 4.2 (iii) and (5.1), we have

$$
\begin{aligned}
\left\|l_{m}(\cdot)\right\|_{r} & \leq \frac{1}{\pi} \int_{0}^{\pi}\|\phi(\eta)\|_{r}\left|K_{m}^{\Delta_{H} N^{p, q}}(\eta)\right| d \eta \\
& \leq \frac{2}{\pi} \int_{0}^{\pi} w_{j}(g, \eta)_{r}\left|K_{m}^{\Delta_{H} N^{p, q}}(\eta)\right| d \eta .
\end{aligned}
$$

Using Hölder's inequality and definition of Besov space, we get

$$
\begin{aligned}
\left\|l_{m}(\cdot)\right\|_{r} & \leq 2\left\{\int_{0}^{\pi}\left(\left|K_{m}^{\Delta_{H} N^{p, q}}(\eta)\right| \eta^{\lambda+q^{-1}}\right)^{q /(q-1)} d \eta\right\}^{1-q^{-1}} \times\left\{\int_{0}^{\pi}\left(\frac{w_{j}(g, \eta)_{r}}{\eta^{\lambda+q^{-1}}}\right)^{q} d \eta\right\}^{q^{-1}} \\
& =O(1)\left\{\int_{0}^{\pi}\left(\left|K_{m}^{\Delta_{H} N^{p, q}}(\eta)\right| \eta^{\lambda+q^{-1}}\right)^{q /(q-1)} d \eta\right\}^{1-q^{-1}} \\
& =O(1)\left\{\left(\int_{0}^{\frac{1}{m+1}}+\int_{\frac{1}{m+1}}^{\pi}\right)\left(\left|K_{m}^{\Delta_{H} N^{p, q}}(\eta)\right| \eta^{\lambda+q^{-1}}\right)^{q /(q-1)} d \eta\right\}^{1-q^{-1}} \\
& :=O(1)\left[I_{1}+I_{2}\right] .
\end{aligned}
$$

Using Lemma 4.1 for $0 \leq \eta \leq \frac{1}{m+1}$, we get

$$
\begin{aligned}
I_{1} & =\left\{\int_{0}^{\frac{1}{m+1}}\left(\left|K_{m}^{\Delta_{H} N^{p, q}}(\eta)\right| \eta^{\lambda+q^{-1}}\right)^{q /(q-1)} d \eta\right\}^{1-q^{-1}} \\
& =O(m+1)\left\{\int_{0}^{\frac{1}{m+1}}\left(\eta^{\lambda+q^{-1}}\right)^{\frac{q}{q-1}} d \eta\right\}^{1-q^{-1}}
\end{aligned}
$$




$$
\begin{aligned}
& =O(m+1)\left\{\int_{0}^{\frac{1}{m+1}} \eta^{\frac{q}{q-1}\left(\lambda+q^{-1}\right)} d \eta\right\}^{1-q^{-1}} \\
& =O\left((m+1)^{-\lambda}\right) .
\end{aligned}
$$

By using Lemma 4.1 for $\frac{1}{m+1} \leq \eta \leq \pi$, we get

$$
\begin{aligned}
I_{2} & =\left\{\int_{\frac{1}{m+1}}^{\pi}\left(\left|K_{m}^{\Delta_{H} N^{p, q}}(\eta)\right| \eta^{\lambda+q^{-1}}\right)^{q /(q-1)} d \eta\right\}^{1-q^{-1}} \\
& =O(1)\left\{\int_{\frac{1}{m+1}}^{\pi}\left(\eta^{\lambda+q^{-1}-1}\right)^{q /(q-1)} d \eta\right\}^{1-q^{-1}} \\
& =O(1)\left\{\int_{\frac{1}{m+1}}^{\pi} \eta^{\frac{q}{q-1}\left(\lambda+q^{-1}-1\right)} d \eta\right\}^{1-q^{-1}} \\
& =O(1)\left\{\int_{\frac{1}{m+1}}^{\pi} \eta^{\frac{q}{q-1} \lambda-1} d \eta\right\}^{1-q^{-1}} \\
& =O\left((m+1)^{-\lambda}\right) .
\end{aligned}
$$

From (5.3), (5.4) and (5.5), we have

$$
\left\|l_{m}(\cdot)\right\|_{r}=O\left((m+1)^{-\lambda}\right) .
$$

Now, using generalized Minkowski’s inequality and using Lemma 4.3, we have

$$
\begin{aligned}
\left\|w_{j}\left(l_{m}, \cdot\right)\right\|_{\delta, q} & =\left\{\int_{0}^{\pi}\left(\frac{w_{j}\left(l_{m}, l\right) r}{l^{\delta}}\right)^{q} \frac{d l}{l}\right\}^{q^{-1}} \\
& =\left\{\int_{0}^{\pi}\left(\frac{\left\|\mathcal{L}_{m}(\cdot, l)\right\|_{r}}{l^{\delta}}\right)^{q} \frac{d l}{l}\right\}^{q^{-1}} \\
& =\left\{\int_{0}^{\pi}\left(\frac{1}{2 \pi} \int_{0}^{2 \pi}\left|\mathcal{L}_{m}(z, l)\right|^{r} d z\right)^{q / r} \frac{d l}{l^{\delta q+1}}\right\}^{q^{-1}} \\
& =\left\{\int_{0}^{\pi}\left(\frac{1}{2 \pi} \int_{0}^{2 \pi}\left|\frac{1}{\pi} \int_{0}^{\pi} \Phi(z, l, \eta) K_{m}^{\Delta_{H} N^{p, q}}(\eta) d \eta\right|^{r} d z\right)^{q / r} \frac{d l}{l^{\delta q+1}}\right\}^{q^{-1}} \\
& \leq \frac{1}{\pi}\left[\int_{0}^{\pi}\left(\frac{1}{2 \pi}\right)^{q / r} \times\left\{\int_{0}^{\pi}\left(\int_{0}^{2 \pi}|\Phi(z, l, \eta)|^{r}\left|K_{m}^{\Delta_{H} N^{p, q}}(\eta)\right|^{r} d z\right)^{1 / r} d \eta\right\} \frac{d l}{l^{\delta q+1}}\right]^{q^{-1}} \\
= & \frac{1}{\pi}\left[\int_{0}^{\pi}\left\{\int_{0}^{\pi}\|\Phi(\cdot, l, \eta)\|_{r} \mid K_{m}^{\Delta_{H} N^{p, q}}(\eta) d \eta\right\} \frac{d l}{l \delta q+1}\right]^{q^{-1}}
\end{aligned}
$$




$$
\begin{aligned}
& \leq \frac{1}{\pi} \int_{0}^{\pi}\left|K_{m}^{\Delta_{H} N^{p, q}}(\eta)\right| d \eta\left(\int_{0}^{\pi} \frac{\|\Phi(\cdot, l, \eta)\|_{r}^{q}}{l \delta q} \frac{d l}{l}\right)^{q^{-1}} \\
& =\frac{1}{\pi} \int_{0}^{\pi} \mid K_{m}^{\Delta_{H} N^{p, q}}(\eta) d \eta\left\{\left(\int_{0}^{\eta}+\int_{\eta}^{\pi}\right) \frac{\|\Phi(\cdot, l, \eta)\|_{r}^{q}}{l \delta q} \frac{d l}{l}\right\}^{q^{-1}} \\
& \leq \frac{1}{\pi} \int_{0}^{\pi}\left|K_{m}^{\Delta_{H} N^{p, q}}(\eta)\right| d \eta\left\{\int_{0}^{\eta} \frac{\|\Phi(\cdot, l, \eta)\|_{r}^{q}}{l} \frac{d l}{l}\right\}^{q^{-1}} \\
& \quad+\frac{1}{\pi} \int_{0}^{\pi}\left|K_{m}^{\Delta_{H} N^{p, q}}(\eta)\right| d \eta\left\{\int_{\eta}^{\pi} \frac{\| \Phi\left(\cdot, l, \eta \|_{r}^{q}\right)}{l} \frac{d l}{l}\right\}^{q^{-1}} \\
& =O(1)\left\{\int_{0}^{\pi}\left(\eta^{\lambda-\delta}\left|K_{m}^{\Delta_{H} N^{p, q}}(\eta)\right|\right)^{q /(q-1)} d \eta\right\}^{1-(1 / q)} \\
& +O(1)\left\{\int_{0}^{\pi}\left(\eta^{\lambda-\delta+(1 / q)} \mid K_{m}^{\Delta_{H} N^{p, q}}(\eta)\right)^{q /(q-1)} d \eta\right\}^{1-(1 / q)} \\
& :=O(1)\left(J_{1}+J_{2}\right) .
\end{aligned}
$$

Since $(x+y)^{r} \leq x^{r}+y^{r}$ for positive $x, y$ and $0<r \leq 1$, then

$$
\begin{aligned}
J_{1} & =\left\{\int_{0}^{\pi}\left(\eta^{\lambda-\delta}\left|K_{m}^{\Delta_{H} N^{p, q}}(\eta)\right|\right)^{q /(q-1)} d \eta\right\}^{1-q^{-1}} \\
& =\left\{\left(\int_{0}^{1 /(m+1)}+\int_{1 /(m+1)}^{\pi}\right)\left(\eta^{\lambda-\delta}\left|K_{m}^{\Delta_{H} N^{p, q}}(\eta)\right|\right)^{q /(q-1)}\right\}^{1-q^{-1}} \\
& \leq\left\{\int_{0}^{1 /(m+1)}\left(\eta^{\lambda-\delta}\left|K_{m}^{\Delta_{H} N^{p, q}}(\eta)\right|\right)^{q /(q-1)} d \eta\right\}^{1-q^{-1}} \\
& +\left\{\int_{1 /(m+1)}^{\pi}\left(\eta^{\lambda-\delta}\left|K_{m}^{\Delta_{H} N^{p, q}}(\eta)\right|\right)^{q /(q-1)} d \eta\right\}^{1-q^{-1}} \\
& =I_{11}+I_{12} .
\end{aligned}
$$

Using Lemma 4.1 for $0 \leq \eta \leq \frac{1}{m+1}$, we have

$$
\begin{aligned}
I_{11} & =\left\{\int_{0}^{1 /(m+1)}\left(\eta^{\lambda-\delta}\left|K_{m}^{\Delta_{H} N^{p, q}}(\eta)\right|\right)^{q /(q-1)} d \eta\right\}^{1-q^{-1}} \\
& =O(m+1)\left\{\int_{0}^{1 /(m+1)} \eta^{\frac{q}{q-1}(\lambda-\delta)} d \eta\right\}^{1-q^{-1}} \\
& =O(m+1)\left\{\int_{0}^{1 /(m+1)} \eta^{\frac{q}{q-1}(\lambda-\delta+1-(1 / q))-1} d \eta\right\}^{1-q^{-1}} \\
& =O\left(\frac{1}{(m+1)^{\lambda-\delta-(1 / q)}}\right) .
\end{aligned}
$$


Using Lemma 4.1 for $\frac{1}{m+1} \leq \eta \leq \pi$, we have

$$
\begin{aligned}
I_{12} & =\left\{\int_{1 /(m+1)}^{\pi}\left(\eta^{\lambda-\delta}\left|K_{m}^{\Delta_{H} N^{p, q}}(\eta)\right|\right)^{q /(q-1)} d \eta\right\}^{1-q^{-1}} \\
& =O(1)\left\{\int_{1 /(m+1)}^{\pi}\left(\eta^{\lambda-\delta-1}\right)^{\frac{q}{q-1}} d \eta\right\}^{1-q^{-1}} \\
& =O(1)\left\{\int_{1 /(m+1)}^{\pi} \eta^{\frac{q}{q-1}(\lambda-\delta-1)} d \eta\right\}^{1-q^{-1}} \\
& =O(1)\left\{\int_{1 /(m+1)}^{\pi} \eta^{\frac{q}{q-1}(\lambda-\delta-(1 / q))-1} d \eta\right\}^{1-q^{-1}} \\
& =O\left(\frac{1}{(m+1)^{\lambda-\delta-(1 / q)}}\right) .
\end{aligned}
$$

From (5.8), (5.9) and (5.10), we have

$$
\begin{aligned}
J_{1} & :=I_{11}+I_{12} \\
& =O\left(\frac{1}{(m+1)^{\lambda-\delta-(1 / q)}}\right) .
\end{aligned}
$$

Now,

$$
\begin{aligned}
J_{2} & =\left\{\int_{0}^{\pi}\left(\eta^{\lambda-\delta+1 / q}\left|K_{m}^{\Delta_{H} N^{p, q}}(\eta)\right|\right)^{q /(q-1)} d \eta\right\}^{1-q^{-1}} \\
& =\left[\left\{\int_{0}^{1 /(m+1)}+\int_{1 /(m+1)}^{\pi}\right\}\left(\eta^{\lambda-\delta+(1 / q)}\left|K_{m}^{\Delta_{H} N^{p, q}}(\eta)\right|\right)^{q /(q-1)} d \eta\right]^{1-q^{-1}} \\
& :=J_{11}+J_{12}
\end{aligned}
$$

Using Lemma 4.1 for $0 \leq \eta \leq \frac{1}{m+1}$, we have

$$
\begin{aligned}
J_{11} & =\left\{\int_{0}^{1 /(m+1)}\left(\eta^{\lambda-\delta+(1 / q)}\left|K_{m}^{\Delta_{H} N^{p, q}}(\eta)\right|\right)^{q /(q-1)} d \eta\right\}^{1-q^{-1}} \\
& =O(m+1)\left\{\int_{0}^{1 /(m+1)}\left(\eta^{\lambda-\delta+(1 / q)}\right)^{q /(q-1)} d \eta\right\}^{1-q^{-1}} \\
& =O(m+1)\left\{\int_{0}^{1 /(m+1)} \eta^{\frac{q}{q-1}(\lambda-\delta+1)-1} d \eta\right\}^{1-q^{-1}} \\
& =O\left(\frac{1}{(m+1)^{\lambda-\delta}}\right) .
\end{aligned}
$$


Using Lemma 4.1 for $\frac{1}{m+1} \leq \eta \leq \pi$, we have

$$
\begin{aligned}
J_{12} & =\left\{\int_{1 /(m+1)}^{\pi}\left(\eta^{\lambda-\delta+(1 / q)}\left|K_{m}^{\Delta_{H} N^{p, q}}(\eta)\right|\right)^{q /(q-1)} d \eta\right\}^{1-q^{-1}} \\
& =O(1)\left\{\int_{1 /(m+1)}^{\pi}\left(\eta^{\lambda-\delta+(1 / q)-1}\right)^{q /(q-1)} d \eta\right\}^{1-q^{-1}} \\
& =O(1)\left\{\int_{1 /(m+1)}^{\pi} \eta^{\frac{q}{q-1}(\lambda-\delta)-1} d \eta\right\}^{1-q^{-1}} \\
& =O\left(\frac{1}{(m+1)^{\lambda-\delta}}\right) .
\end{aligned}
$$

From (5.12), (5.13) and (5.14), we have

$$
\begin{aligned}
J_{2} & :=J_{11}+J_{12} \\
& =O\left(\frac{1}{(m+1)^{\lambda-\delta}}\right) .
\end{aligned}
$$

From (5.7), (5.11) and (5.15), we get

$$
\begin{aligned}
\left\|w_{j}\left(l_{m}, \cdot\right)\right\|_{\delta, q} & =O(1)\left(J_{1}+J_{2}\right) \\
& =O(1)\left[O\left(\frac{1}{(m+1)^{\lambda-\delta-(1 / q)}}\right)+O\left(\frac{1}{(m+1)^{\lambda-\delta}}\right)\right] .
\end{aligned}
$$

From (5.2), (5.6) and (5.16), we get

$$
\begin{aligned}
\left\|l_{m}(\cdot)\right\|_{B_{q}^{\delta}\left(L^{r}\right)} & =\left\|l_{m}(\cdot)\right\|_{r}+\left\|w_{j}\left(l_{m}, \cdot\right)\right\|_{\delta, q} \\
& =O(m+1)^{-\lambda}+O\left(\frac{1}{(m+1)^{\lambda-\delta-(1 / q)}}\right)+O\left(\frac{1}{(m+1)^{\lambda-\delta}}\right) .
\end{aligned}
$$

This completes the proof of case I.

Case II : For $q=\infty, 0 \leq \delta<\lambda<2$.

We have

$$
\left\|l_{m}(\cdot)\right\|_{B_{\infty}^{\delta}\left(L^{r}\right)}=\left\|l_{m}(\cdot)\right\|_{r}+\left\|w_{j}\left(l_{m}, \cdot\right)\right\|_{\delta, \infty} .
$$

Using (1.6), we have

$$
\begin{aligned}
\left\|l_{m}(\cdot)\right\|_{r} & \leq 2 \int_{0}^{\pi} w_{j}(g, \eta)_{r}\left|K_{m}^{\Delta_{H} N^{p, q}}(\eta)\right| d \eta \\
& =O(1)\left\{\int_{0}^{1 /(m+1)} \eta^{\lambda}\left|K_{m}^{\Delta_{H} N^{p, q}}(\eta)\right| d \eta+\int_{1 /(m+1)}^{\pi} \eta^{\lambda}\left|K_{m}^{\Delta_{H} N^{p, q}}(\eta)\right| d \eta\right\}
\end{aligned}
$$




$$
:=O(1)\left(I_{2}+J_{2}\right)
$$

Using Lemma 4.1 for $0 \leq \eta \leq \frac{1}{m+1}$, we get

$$
\begin{aligned}
I_{2} & =\int_{0}^{1 /(m+1)} \eta^{\lambda}\left|K_{m}^{\Delta_{H} N^{p, q}}(\eta)\right| d \eta \\
& =O(m+1) \int_{0}^{1 /(m+1)} \eta^{\lambda} d \eta \\
& =O(m+1)^{-\lambda} .
\end{aligned}
$$

Again, using Lemma 4.1 for $\frac{1}{m+1} \leq \eta \leq \pi$, we get

$$
\begin{aligned}
J_{2} & =\int_{1 /(m+1)}^{\pi} \eta^{\lambda}\left|K_{m}^{\Delta_{H} N^{p, q}}(\eta)\right| d \eta \\
& =O(1) \int_{1 /(m+1)}^{\pi} \eta^{\lambda-1} d \eta \\
& =O(m+1)^{-\lambda} .
\end{aligned}
$$

From (5.18), (5.19) and (5.20), we get

$$
\begin{aligned}
\left\|l_{m}(\cdot)\right\|_{r} & =O(1)\left(I_{2}+J_{2}\right) \\
& =O(m+1)^{-\alpha} .
\end{aligned}
$$

Using generalized Minkowski’s inequality and Lemma 4.4, we get

$$
\begin{aligned}
\| w_{j} & \left(l_{m}, \cdot\right) \|_{\delta, \infty} \\
= & \sup _{l>0}\left(l^{-\delta} w_{j}\left(l_{m}, l\right)_{r}\right) \\
= & \sup _{l>0}\left(l^{-\delta}\left\|\mathcal{L}_{m}(\cdot, l)\right\|_{r}\right) \\
= & \sup _{l>0}\left[l^{-\delta}\left(\frac{1}{2 \pi} \int_{0}^{2 \pi}\left|\int_{0}^{\pi} K_{m}^{\Delta_{H} N^{p, q}}(\eta) \phi(z, l, \eta) d \eta\right|^{r} d z\right)^{1 / r}\right] \\
\leq & \sup _{l>0}\left[\frac{l^{-\delta}}{\pi}\left(\frac{1}{2 \pi}\right)^{1 / r} \int_{0}^{\pi}\left\{\int_{0}^{2 \pi}\left|K_{m}^{\Delta_{H} N^{p, q}}(\eta)\right|^{r}|\phi(z, l, \eta)|^{r} d z\right\}^{1 / r} d \eta\right] \\
= & \sup _{l>0}\left[\frac{l^{-\delta}}{\pi} \int_{0}^{\pi}\|\phi(\cdot, l, \eta)\|_{r}\left|K_{m}^{\Delta_{H} N^{p, q}}(\eta)\right| d \eta\right] \\
= & \frac{1}{\pi} \int_{0}^{\pi}\left(\sup _{l>0} l^{-\delta}\|\Phi(\cdot, l, \eta)\|_{r}\right)\left|K_{m}^{\Delta_{H} N^{p, q}}(\eta)\right| d \eta \\
= & O(1) \int_{0}^{\pi} \eta^{\lambda-\delta}\left|K_{m}^{\Delta_{H} N^{p, q}}(\eta)\right| d \eta
\end{aligned}
$$




$$
\begin{aligned}
& =O(1)\left[\int_{0}^{1 /(m+1)} \eta^{\lambda-\delta}\left|K_{m}^{\Delta_{H} N^{p, q}}(\eta) d \eta+\int_{1 /(m+1)}^{\pi} \eta^{\lambda-\delta}\right| K_{m}^{\Delta_{H} N^{p, q}}(\eta) \mid d \eta\right] \\
& =O(1)\left[I_{3}+J_{3}\right] .
\end{aligned}
$$

Using Lemma 4.1 for $0 \leq \eta \leq \frac{1}{m+1}$, we get

$$
\begin{aligned}
I_{3} & =\int_{0}^{1 /(m+1)} \eta^{\lambda-\delta}\left|K_{m}^{\Delta_{H} N^{p, q}}(\eta)\right| d \eta \\
& =O(m+1) \int_{0}^{1 /(m+1)} \eta^{\lambda-\delta} d \eta \\
& =O\left(\frac{1}{(m+1)^{\lambda-\delta}}\right) .
\end{aligned}
$$

Using Lemma 4.1 for $\frac{1}{m+1} \leq \eta \leq \pi$, we get

$$
\begin{aligned}
J_{3} & =\int_{1 /(m+1)}^{\pi} \eta^{\lambda-\delta}\left|K_{m}^{\Delta_{H} N^{p, q}}(\eta)\right| d \eta \\
& =O(1) \int_{1 /(m+1)}^{\pi} \eta^{\lambda-\delta-1} d \eta \\
& =O\left(\frac{1}{(m+1)^{\lambda-\delta}}\right) .
\end{aligned}
$$

From (5.22), (5.23) and (5.24), we get

$$
\begin{aligned}
\left\|w_{j}\left(l_{m}, \cdot\right)\right\|_{\delta, \infty} & =O(1)\left[I_{3}+J_{3}\right] \\
& =O\left(\frac{1}{(m+1)^{\lambda-\delta}}\right) .
\end{aligned}
$$

From (5.17), (5.21) and (5.25), we have

$$
\begin{aligned}
\left\|l_{m}(\cdot)\right\|_{B_{\infty}^{\beta}\left(L^{r}\right)} & =\left\|l_{m}(\cdot)\right\|_{r}+\left\|w_{j}\left(l_{m}, \cdot\right)\right\|_{\delta, \infty} \\
& =O(m+1)^{-\lambda}+O\left(\frac{1}{(m+1)^{\lambda-\delta}}\right) .
\end{aligned}
$$

This completes the proof of case II.

\section{Corollary}

The following corollary are derived from our main theorem.

Corollary 6.1. If $q_{m}=1 \forall m$, then $\Delta_{H} N^{p, q}$ means reduces to $\Delta_{H} N^{p_{m}}$ means and the best approximation of $g \in B_{q}^{\lambda}\left(L^{r}\right)$ space by $\Delta_{H} N^{p_{m}}$ means of Fourier series is

$\left\|t_{m}^{\Delta_{H} N^{p_{m}}}(z)-g(z)\right\|= \begin{cases}O(m+1)^{-\lambda}+O\left(\frac{1}{(m+1)^{\lambda-\delta-(1 / q)}}\right)+O\left(\frac{1}{(m+1)^{\lambda-\delta}}\right) & ; 1<q<\infty \\ O(m+1)^{-\lambda}+O\left(\frac{1}{(m+1)^{\lambda-\delta}}\right) & ; q=\infty .\end{cases}$ 
Corollary 6.2. If $p_{m}=1 \forall m$, then $\Delta_{H} N^{p, q}$ reduces to $\Delta_{H} \tilde{N}^{q_{m}}$ means and the best approximation of $g \in B_{q}^{\lambda}\left(L^{r}\right)$ space by $\Delta_{H} \tilde{N}^{q_{m}}$ means of Fourier series is

$\left\|t_{m}^{\Delta_{H} \tilde{N}^{q_{m}}}(z)-g(z)\right\|= \begin{cases}O(m+1)^{-\lambda}+O\left(\frac{1}{(m+1)^{\lambda-\delta-(1 / q)}}\right)+O\left(\frac{1}{(m+1)^{\lambda-\delta}}\right) & ; 1<q<\infty \\ O(m+1)^{-\lambda}+O\left(\frac{1}{(m+1)^{\lambda-\delta}}\right) & ; q=\infty .\end{cases}$

Corollary 6.3. If $p_{m}=\left(\begin{array}{c}m+\alpha-1 \\ \alpha-1\end{array}\right) \alpha>0$, and $q_{m}=1 \forall m$, then $\Delta_{H} N^{p, q}$ means reduces to $\Delta_{H} C_{\alpha}$ means of and the best approximation of $g \in B_{q}^{\lambda}\left(L^{r}\right)$ space by $\Delta_{H} C_{\alpha}$ means of Fourier series is $\left\|t_{m}^{\Delta_{H} C_{\alpha}}(z)-g(z)\right\|= \begin{cases}O(m+1)^{-\lambda}+O\left(\frac{1}{(m+1)^{\lambda-\delta-(1 / q)}}\right)+O\left(\frac{1}{(m+1)^{\lambda-\delta}}\right) & ; 1<q<\infty \\ O(m+1)^{-\lambda}+O\left(\frac{1}{(m+1)^{\lambda-\delta}}\right) & ; q=\infty .\end{cases}$ Corollary 6.4. If $h_{m, j}=\left\{\begin{array}{ll}\left(\begin{array}{c}m \\ j\end{array}\right) \frac{q^{m-j}}{(1+q)^{m}}, & \text { if } 0 \leq j \leq m, \\ 0, & \text { if } j>m .\end{array}\right.$, then $\Delta_{H} N^{p, q}$ means reduces to $E_{q} N^{p, q}$ means and the best approximation of $g \in B_{q}^{\lambda}\left(L^{r}\right)$ space by $E_{q} N^{p, q}$ means of Fourier series is $\left\|t_{m}^{E_{q} N^{p, q}}(z)-g(z)\right\|= \begin{cases}O(m+1)^{-\lambda}+O\left(\frac{1}{(m+1)^{\lambda-\delta-(1 / q)}}\right)+O\left(\frac{1}{(m+1)^{\lambda-\delta}}\right) & ; 1<q<\infty \\ O(m+1)^{-\lambda}+O\left(\frac{1}{(m+1)^{\lambda-\delta}}\right) & ; q=\infty .\end{cases}$ Corollary 6.5. If $h_{m, j}=\left\{\begin{array}{ll}\frac{1}{m+1}, & \text { if } 0 \leq j \leq m, \\ 0, & \text { if } j>m .\end{array}\right.$, then $\Delta_{H} N^{p, q}$ means reduces to $C_{1} N^{p, q}$ means and the best approximation of $g \in B_{q}^{\lambda}\left(L^{r}\right)$ space by $C_{1} N^{p, q}$ means of Fourier series is $\left\|t_{m}^{C_{1} N^{p, q}}(z)-g(z)\right\|= \begin{cases}O(m+1)^{-\lambda}+O\left(\frac{1}{(m+1)^{\lambda-\delta-(1 / q)}}\right)+O\left(\frac{1}{(m+1)^{\lambda-\delta}}\right) & ; 1<q<\infty \\ O(m+1)^{-\lambda}+O\left(\frac{1}{(m+1)^{\lambda-\delta}}\right) & ; q=\infty .\end{cases}$

\section{Particular cases}

(i) In view of Remark 2 (i) and 3 (ii), our result becomes a particular case of [10].

(ii) In view of Remark 2 (i) and 3 (iv), our result becomes a particular case of [26].

(iii) In view of Remark 2 (ii) and 3 (v), our result becomes a particular case of [27].

\section{Acknowledgements}

First author expresses his gratitude towards his mother for her blessings. The first author also expresses his gratitude towards his father in heaven whose soul is always guiding and encouraging 
him. The first author is also thankful to Council of Scientific and Industrial Research, Government of India for his support to the work under the project 25/(0225)/13/EMR-II. Second author is thankful to her grandfather for his encouragement.

\section{References}

[1] A. DeVore Ronald and G. Lorentz, Costructive Approximation, Springer-Verlay, Berlin Heidelberg, New York, (1993).

[2] A. Zygmund, Smooth functions, Duke Math. Journal, 12, 47 - 56, (1945).

[3] A. Zygmund, Trigonometric series, 2nd rev. ed., I, Cambridge Univ. Press, Cambridge, 51 (1968).

[4] B. E. Rhoades, On the degree of approximation of functions belonging to the weighted $\left(L^{p}, \xi(t)\right)$-class by Hausdorff means, Tamkang J. Math., 32(4), $305-314$, (2001).

[5] E. C. Titchmarsh, The Theory of functions, Second Edition, Oxford University Press, p. 403, (1939).

[6] F. Hausdorff, Summationsmethoden and Momentfolgen, Math. Z., 9, I: 74-109, II : 280-289, (1921).

[7] G. Alexits, Convergence problems of orthogonal series, Translated from the German by I. Földer. International Series of Monographs in Pure and Applied Mathematics, vol. 20. Pergamom, New York (1961).

[8] G. Das, A. Nath and B. K. Ray, An estimate of the rate of convergence of Fourier series in the generalized Hölder metric, Analysis and Applications, 43 - 60, (2002).

[9] G. Das, T. Ghosh and B. K. Ray, Degree of approximation by their Fourier series in generalized Hölder metric, Proc Indian Acad. Sci. Math. Sci., 106, 139 - 153, (1996).

[10] H. K. Nigam and K. Sharma, Approximation of functions belonging to different classes of functions by $(E, 1)\left(N, p_{n}\right)$ product means, Lobachevskii Journal of Mathematics, Vol. 32(4), $345-357,(2011)$.

[11] H. L. Garabedian, Hausdorff matrices, Amer. Math. Monthly, 46(7), 390 - 410, (1939).

[12] H. Mohanty, G. Das and B. K. Ray, Degree of approximation of Fourier series of function in Besov space by $\left(N, p_{n}\right)$ mean, J. Orissa Math. Soc., 30(2), $13-34,(2011)$. 
[13] H. Triebel, Theory of Function spaces, Reprint of 1983 edition. Birhäuser/Springer Basel, (2010).

[14] I. J. Maddox, Elements of functional Analysis, Cambridge Univ. Press, Cambridge, (1970).

[15] L. Leindler, Trigonometric approximation in $L_{p}$-norm, J. Math. Anal. Appl., 302, 129 - 136, (2005).

[16] L. Leindler, A relaxed estimate of the degree of approximation by Fourier series in generalized Hölder metric, Anal. Math., 35 (1), 51 - 60, (2009).

[17] L. McFadden, Absolute Nörlund summability, Duke Math. J., 9, 168 - 207, (1942).

[18] M. L. Mittal, B. E. Rhoades, V. N. Mishra and U. Singh, Using infinite matrices to approximate functions of class Lip $(\alpha, p)$ using trigonometric polynomial, J. Math. Anal. Appl., 326(1), $667-676,(2007)$.

[19] M. L. Mittal, B. E. Rhoades, S. Sonker and U. Singh, Approximation of signals of class Lip $(\alpha, p)$ by linear operators, Appl. Math. Comput., 217(9), 4483 - 4489, (2011).

[20] M. L. Mittal and M. V. Singh, Approximation of signals (functions) by trigonometric polynomials in $L_{p}$-norm, Int. J. Math. Math. Sci., Article ID : 267383, (2014).

[21] R. N. Mohapatra and P. Chandra, Degree of approximation of functions in the Hölder metric, Acta Math. Hung., 41(1-2), $67-76,(1983)$.

[22] P. Chandra, On the generalized Fejér means in the metric of Hölder space, Math. Nachr. 109, $39-45,(1982)$.

[23] P. Chandra, Trigonometric approximation of functions in $L_{p}$-norm, J. Math. Anal. Appl., 275, $13-26,(2002)$.

[24] P. Wojtaszczyk, A Mathematical Introduction to Wavelets, London Mathematical Society Student Texts, Cambridge University Press, New York, Vol. 37, (1997).

[25] E. S. Quade, Trigonometric approximation in the mean, Duke Math. J., 3(3), 529 - 543, (1937).

[26] R. Singh and S. S. Thakur, Degree of approximation of continuous functions by $(E, q)(C, \delta)$ means, Gen. Math. Notes, Vol. 11(2), 12 - 19, (2012).

[27] S. Lal, Approximation of functions belonging to the generalized Lipschitz class by $C^{1} \cdot N_{p}$ summability method of Fourier series, Appl. Math. Comp., 209, 346 - 350, (2009). 
[28] S. Prössdorf, Zor Konvergenz der Fourierreihen Hölder stetiger, Funktionen. Math Nachr., 69, $7-14,(1975)$.

Hare Krishna Nigam Department of Mathematics, Central University of South Bihar, Gaya 824236 (Bihar), India

E-mail: hknigam@cusb.ac.in

Supriya Rani Department of Mathematics, Central University of South Bihar, Gaya - 824236 (Bihar), India

E-mail: supriya@cusb.ac.in 